MATHEMATICS OF COMPUTATION

Volume 77, Number 261, January 2008, Pages 589-607

S $0025-5718(07) 02017-0$

Article electronically published on September 12, 2007

\title{
ON SCHÖNHAGE'S ALGORITHM AND SUBQUADRATIC INTEGER GCD COMPUTATION
}

\author{
NIELS MÖLLER
}

\begin{abstract}
We describe a new subquadratic left-to-right GCD algorithm, inspired by Schönhage's algorithm for reduction of binary quadratic forms, and compare it to the first subquadratic GCD algorithm discovered by Knuth and Schönhage, and to the binary recursive GCD algorithm of Stehle and Zimmermann. The new GCD algorithm runs slightly faster than earlier algorithms, and it is much simpler to implement. The key idea is to use a stop condition for HGCD that is based not on the size of the remainders, but on the size of the next difference. This subtle change is sufficient to eliminate the back-up steps that are necessary in all previous subquadratic left-to-right GCD algorithms. The subquadratic GCD algorithms all have the same asymptotic running time, $O\left(n(\log n)^{2} \log \log n\right)$.
\end{abstract}

\section{INTRODUCTION}

In this paper, we describe four subquadratic GCD algorithms: Schönhage's algorithm from 1971, Stehlé's and Zimmermann's binary recursive GCD, a hitherto unpublished GCD algorithm discovered by Schönhage in 1987, and a novel GCD algorithm that uses similar ideas in a HGCD framework. The algorithms are compared with respect to running time and implementation complexity. The new algorithm is slightly faster than all the earlier algorithms, and much simpler to implement.

The paper is organized as follows: First we review the development of integer GCD algorithms in recent years. Section 2 describes the general structure and flavor of the subquadratic GCD algorithms, the idea of using a half-GCD function, and the resulting asymptotic running time. In Section 3, we briefly describe one variant of Schönhage's 1971 algorithm, and in Section 4 , we describe the binary recursive GCD algorithm. The objective of these two sections is to provide sufficient details so that the new algorithm can be compared to earlier algorithms; we define the corresponding half-GCD functions, but we don't provide correctness proofs or detailed analysis.

Section 5 describes a GCD algorithm modeled on Schönhage's algorithm for reduction of binary quadratic forms [8], and in Section 6, this algorithm is reorganized into half-GCD form, resulting in a novel GCD algorithm. Section 7 describes the implementation of the different GCD algorithms, their running times, and code complexity.

Received by the editor November 19, 2005.

2000 Mathematics Subject Classification. Primary 11A05, 11 Y16.

(C)2007 Niels Möller 
1.1. History. Euclid's algorithm for computation of the greatest common divisor is one of the oldest algorithms known. For inputs of size $n$, this algorithm runs in time $O\left(n^{2}\right)$. Since the total size of the remainders is $O\left(n^{2}\right)$, no algorithm which computes all the remainders can run asymptotically faster than this.

Lehmer's algorithm from 1938 cuts the running time of Euclid's algorithm by a constant factor [4. Lehmer's algorithm computes the initial part of the quotient sequence using single or double precision arithmetic on the most significant words of the input, and applies several quotients at once to the multi-precision numbers. Precise conditions for how many correct quotients can be computed in this way were given by Tudor Jebelean in 1995 [2]

Euclid's and Lehmer's algorithms essentially work from the most significant end of the input numbers, and compute a sequence of quotients. A different method, binary GCD, was discovered by Stein in 1961 [11. This algorithm starts from the least significant end. It divides out powers of two, and repeatedly uses subtraction to cancel the least significant bits. In 1994, Jonathan Sorenson described a $k$-ary reduction which gains speed over the basic binary algorithm by doing more work on just the least significant words 9] (cf., Lehmer's algorithm which does more of the work on the most significant one or two words), and this method was improved further in Kenneth Weber's accelerated GCD algorithm from 1995 13.

Up to the twentieth century, GCD algorithms with quadratic running time have been dominating in practice, and it has been the slowest of the algorithms for basic arithmetic. E.g., subquadratic multiplication using the Karatsuba method has been known since the 1960s, and multiplication based on FFT was discovered by several people (Strassen, Schönhage and Pollard) in the early 1970s, and these methods are also in wide use.

The first subquadratic algorithm for GCD computation was described by Donald Knuth in 1970 [3], and it was improved by Schönhage in the next year [7]. These algorithms are essentially algorithms for computing the continued fraction representation of a rational (or even real) number. One crucial observation is that even if the total length of the remainders produced by Euclid's algorithm is quadratic, the total length of the quotients is only $O(n)$. Schönhage's algorithm uses divideand-conquer to recursively compute the quotient sequence. A clear description of the algorithm details, for both the integer and polynomial case, can be found in a paper by Klaus Thull and Chee K. Yap [12. A similar algorithm is described tersely in 6].

Schönhage's 1971 algorithm is straightforward to apply to polynomial GCD, but, to quote [12]: "The integer HGCD algorithm turns out to be rather intricate". Both analysis and actual implementation are quite difficult and error prone. For the same reasons, the algorithm is seldom spelled out in detail in textbooks, and when it is, it has been plagued by errors.

In 1987, Schönhage worked out a related algorithm that does not suffer from the same intricacies, but the algorithm was not published at that time. For the next decade, the new algorithm was known to Schönhage and a few students and correspondents, but not generally known to other researchers or to authors of bignum packages [5, 14.

In a paper from 1991, on the reduction of binary quadratic forms $[8$, Schönhage applies the same ideas to a different problem. This algorithm can be translated, step 
by step, into a GCD algorithm that probably is equivalent to Schönhage's unpublished 1987 algorithm. Another application of the same ideas to a different algebraic context, is Weilert's algorithm for GCD computations on Gaussian integers [15. In this paper, however, we will consider only the classic case of integer GCD.

Since the recursive left-to-right algorithm from 1971 seemed so difficult in practice, Stehlé and Zimmermann investigated if one could use a similar divide-andconquer technique in a right-to-left fashion. In 2004 they published a binary recursive GCD algorithm [10, which is significantly simpler. It is related to the binary GCD algorithm in roughly the same way that Schönhage's algorithm is related to Euclid's algorithm.

As far as the author is aware, there's still no published reference for Schönhage's algorithm from 1987. Section 5 in this paper intends to fill that gap.

1.2. Notation. Sizes of numbers will be important, so we introduce the notation $\# x$ for the bit size of $x$. Define $\# x=\left\lceil\log _{2}(1+|x|)\right\rceil$; then for $x \neq 0, \# x=s$ means that $2^{s-1} \leq|x|<2^{s}$. When \# is applied to a vector or matrix, it denotes the maximum bit size of the elements. Occasionally, we also need the minimum bit size, for which we use the notation $\#(x, y)=\min (\# x, \# y)$.

For the binary algorithm, we use the notation $v(x)$ for the number of zeros at the least significant end of $x$. More precisely, $v(0)=\infty$, and $v(x)=k$ if $2^{k}$ divides $x$ and $2^{-k} x$ is odd.

When comparing matrices, we use the partial order given by elementwise comparison. In particular, $M \geq 0$ means that the elements of $M$ are non-negative. $I$ denotes the identity matrix. For compactness, column vectors are sometimes written as $\left(x_{1} ; x_{2}\right)$ and $2 \times 2$-matrices as $\left(a_{11}, a_{12} ; a_{21}, a_{22}\right)$. All matrices have only integer elements.

\section{General structure of subquadratic GCD Algorithms}

In general, subquadratic GCD uses a divide-and-conquer strategy, based on the observation that we can do a significant fraction of the work by examining only half of the input. The differences between algorithms is in the precise definition of which fraction of the work is done, and which half of the input is used.

We use a function $\operatorname{HGCD}(a, b)$ which takes two $n$-bit numbers as input, and returns two smaller numbers $\alpha, \beta$, of size roughly $n / 2$, and a transformation matrix $M$ whose elements also are roughly of size $n / 2$. The idea is that the smaller numbers $\alpha, \beta$ should have the same GCD as $a, b$, and that the transformation $M$ should be relevant not only for $a, b$, but for some larger numbers that $a, b$ were extracted from.

The HGCD function is computed recursively, using an algorithm of the form shown in Figure 1. The function $\operatorname{Split}(A, p)$ splits $A$ into one piece $a$ consisting of $p$ bits, and a remaining piece $A^{\prime}$. The function $\operatorname{ADJust}\left(\alpha, \beta, M, A^{\prime}, B^{\prime}\right)$ applies $M$ to the parts $A^{\prime}$ and $B^{\prime}$ that were not involved in the previous HGCD call, and combines with the values $\alpha$ and $\beta$ that were returned from that call.

With a fast implementation of HGCD, a fast GCD algorithm can be written as in Figure 2. Each round through the loop reduces the size of $A$ and $B$ by a factor $3 / 4$, and GCD-BASE should be some good quadratic GCD-algorithm, which is used once the numbers are small enough. We will focus on the HGCD algorithm, since GCD is comparatively trivial. 


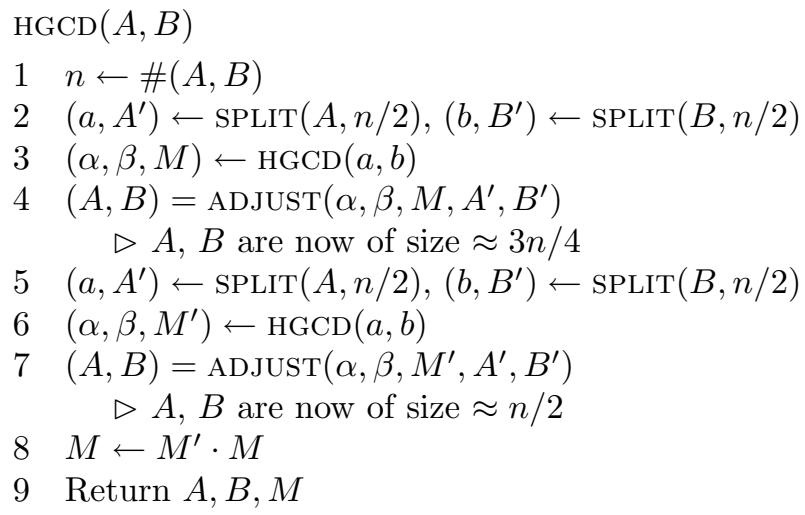

Figure 1. General structure of subquadratic HGCD.

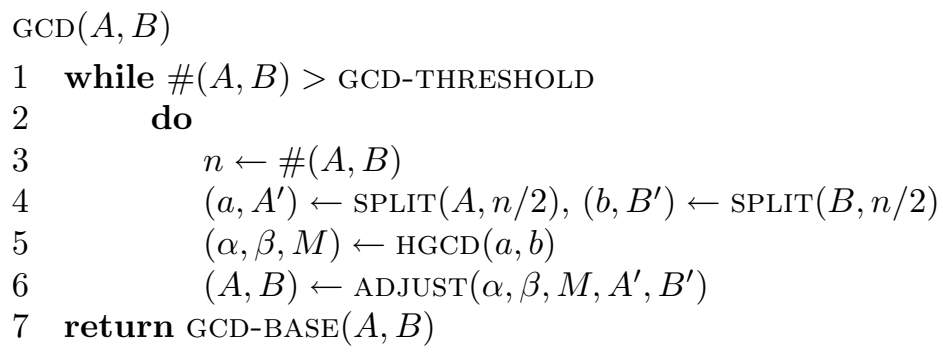

Figure 2. Subquadratic GCD based on HGCD.

2.1. Asymptotic running time. Let $T(n)$ denote the maximum running time of HGCD, with inputs of size $n$, and let $\mu(n)$ denote the maximum time for multiplying or dividing two $n$-bit numbers. The SPLIT function takes $O(n)$ time. The matrix multiplication at line 8 of HGCD corresponds to a small number of scalar multiplications. We haven't yet specified what ADJUST does, but let's assume that it performs a bounded number of multiplications and divisions of $n$-bit numbers. Then it follows that $T(n) \leq 2 T(n / 2)+c \mu(n)$ for some constant $c$, and this inequality implies

$$
T(n) \leq c \mu(n) \log _{2} n .
$$

When FFT methods are used for multiplication, and Newton's method is used for division 1 , $\mu(n)=O(n \log n \log \log n)$, and then we get the asymptotic running time $T(n)=O\left(n(\log n)^{2} \log \log n\right)$.

The additional loop in the GCD algorithm contributes only a constant factor. One round through the loop takes time $T(n / 2)+c^{\prime} \mu(n)$, and reduces the size by a factor $3 / 4$. Then the total time of GCD is bounded by

$$
2 T(n)+4 c^{\prime} \mu(n) .
$$

Hence, GCD needs asymptotically twice as much time as HGCD with inputs of the same size.

\footnotetext{
${ }^{1}$ In practice, division of large numbers will not contribute much to the running time, since, for random inputs, large quotients are very unlikely.
} 


\section{Classical Schönhage GCD}

Given two numbers $a$ and $b, a>b$, define the quotient sequence $q_{i}$ and the remainders $r_{i}$ as

$$
\begin{aligned}
r_{0} & =a, & r_{1} & =b, \\
q_{i} & =\left\lfloor r_{i-1} / r_{i}\right\rfloor, & r_{i+1} & =r_{i-1}-q_{i} r_{i} .
\end{aligned}
$$

Also define two co-sequences $u_{i}$ and $v_{i}$, such that $r_{i}=u_{i} a+v_{i} b$ :

$$
\begin{aligned}
u_{0} & =1, & v_{0} & =0, \\
u_{1} & =0, & v_{1} & =1, \\
u_{i+1} & =u_{i-1}-q_{i} u_{i}, & v_{i+1} & =v_{i-1}-q_{i} v_{i} .
\end{aligned}
$$

The co-factors are bounded by $\left|u_{k}\right| \leq b / r_{k-1},\left|v_{k}\right| \leq a / r_{k-1}$.

Classical Schönhage GCD uses a HGCD-Q function that computes the first half of the quotient sequence. The HGCD-Q function is used with inputs $a$ and $b$ that are the most significant parts of some larger numbers $A$ and $B$, and we want to ensure that the quotients computed for $a$ and $b$ are correct also for $A$ and $B$. This is similar to Lehmer's algorithm, but we use most significant parts $a$ and $b$ that are larger than just one or two words.

The following criterion guarantees that quotients computed from just the most significant parts of two numbers are in fact valid for the full numbers.

Lemma 1 (Jebelean's criterion). Let $a>b>0$, and let $r_{i}, q_{i}$ be the quotient and remainder sequences as defined above. Assume that the following relations are satisfied for $0 \leq i \leq k$ :

$$
\begin{aligned}
r_{i+1} & \geq \max \left(-u_{i+1},-v_{i+1}\right), \\
r_{i}-r_{i+1} & \geq \max \left(u_{i+1}-u_{i}, v_{i+1}-v_{i}\right) .
\end{aligned}
$$

Then for any $p>0$ and any $A^{\prime}$ and $B^{\prime}$, with $0 \leq A^{\prime}, B^{\prime}<2^{p}$, the sequence $q_{1}, \ldots, q_{k}$ is the initial quotient sequence for the numbers $A=a 2^{p}+A^{\prime}, B=a 2^{p}+B^{\prime}$.

We refer to [2] for the proof. There is also a simplified version of the criterion, using only the size of the remainders.

Lemma 2 (Jebelean's simplified criterion). Let $a>b>0$, and let $r_{i}, q_{i}$ be the remainder sequence. Let $n=\# a$ and $m=\lceil n / 2\rceil$. If $k$ is such that $\# r_{k+2}>m$, then Jebelean's criterion is satisfied for $0 \leq i \leq k$.

Note that $\# r_{k}>m+1$ is necessary but not sufficient.

We can now define precisely what the HGCD-Q function is expected to return. The inputs are the two numbers $a$ and $b$, with $a \geq b>0$ and $n=\# a$. Let $m=\lceil n / 2\rceil$. If $\# b \leq m$ or $\#(a \bmod b) \leq m$, the function fails. Otherwise, it computes the quotient sequence until it encounters the first remainder $r_{k+3}$ that fits in $m$ bits. It then returns $r_{k}, r_{k+1}$ and a matrix $M$ made up from the corresponding co-factors. The matrix elements are bounded by $\# M \leq\lfloor n / 2\rfloor-1$.

It is possible to save a little work by returning also $r_{k+2}$ and $r_{k+3}$, and use Jebelean's full criterion to check if the corresponding quotients are correct, but for simplicity we omit the details of this variation.

The algorithm in Figure 3 computes HGCD-Q recursively. The source of most of the complexity of the algorithm is that we do not have precise control over the size of the remainders after the second recursive call. At line 24 we have $\# r_{0}>m$, but $r_{1}$ 


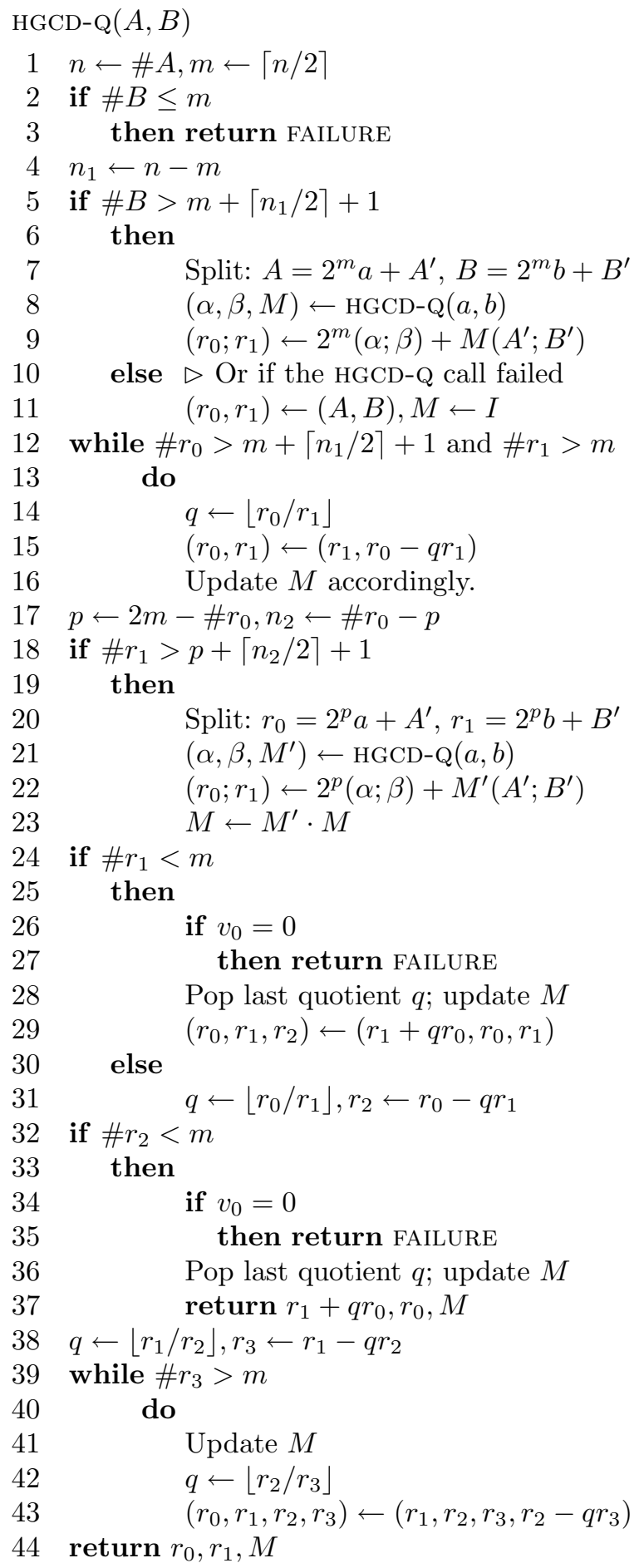

Figure 3. Classical Schönhage GCD 


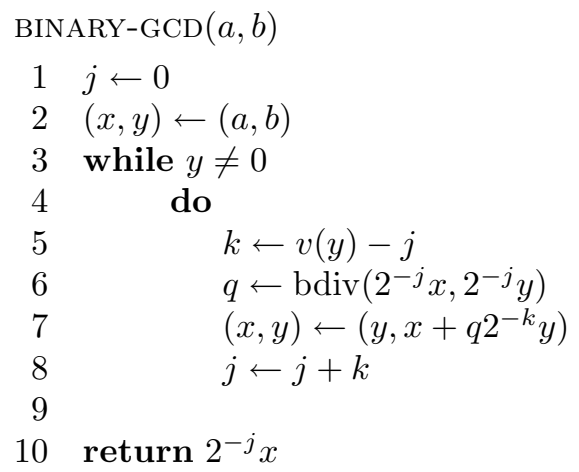

FiguRE 4. Binary GCD

can be smaller. We may have to undo one or two of the divisions performed by the second HGCD-Q call, in order to return two remainders that satisfy the specification; see Section 6.3 for an example where this happens.

How do we find the previous quotient at line 28 and 36? One way is to maintain a quotient stack, storing the complete quotient sequence leading up to the current remainders. The bookkeeping cost for such a stack is $O(n)$ in both space and time. However, the previous quotient can also be recovered directly from $M$. For further details and analysis of this algorithm, we refer to [12.

\section{BinARY RECURSIVE GCD}

Binary GCD works from the least significant end. Instead of the division used in Euclid's algorithm, we use binary division, denoted $\operatorname{bdiv}(a, b)$ and defined as follows. Let $a, b$ be two integers, $a$ odd and $b$ even and non-zero. Let $k=v(b)$, so that $b^{\prime}=2^{-k} b$ is odd. The quotient $q=-a\left(b^{\prime}\right)^{-1}\left(\bmod 2^{k+1}\right)$ satisfies

$$
r=a+q b^{\prime}=0 \quad\left(\bmod 2^{k+1}\right)
$$

and $q$ is uniquely determined modulo $2^{k+1}$. Since $a$ is odd, $q$ is odd too, and can be chosen in the interval $|q|<2^{k}$. Then $2^{-k}|q|<1$, so that

$$
|r|=\left|a+q 2^{-k} b\right| \leq|a|+2^{-k}|q||b|<|a|+|b| .
$$

It is also clear that $q$ depends only on the $k+1$ least significant bits of $a$ and $b^{\prime}$, or on the $2 k+1$ least significant bits of $a$ and $b$.

To apply this to GCD computation, first note that $\operatorname{gcd}(b, r)=2^{k} \operatorname{gcd}(a, b)$. Consider the algorithm in Figure 4. Why does this algorithm terminate? After $n$ rounds through the loop, $j \geq n$, so that $2^{n}$ divides $x$ and $y$. This implies that as long as $y \neq 0$, we must have $|y| \geq 2^{n}$. From this and equation (1), we get

$$
2^{n} \leq|y| \leq F_{n+2} \max (|a|,|b|)
$$

where $F_{n}$ is the $n$th Fibonacci number. These inequalities can hold for only finitely many $n$, hence the algorithm must terminate with $y=0$ after a finite number of steps. The use of quotients in the symmetric interval $-2^{k}<q<2^{k}$ is essential; if one tries to use positive quotients $0<q<2^{k+1}$, the algorithm no longer terminates.

The binary recursive algorithm by Stehlé and Zimmermann uses a function HGCD-B defined as follows. The inputs are a bit size $\ell$ and two numbers $0 \leq a, b<2^{\ell}$, 
with $a$ odd and $b$ even. Let $k=\lfloor(\ell-1) / 2\rfloor$, and consider the remainder sequence $r_{i}$ formed by repeated use of bdiv. For some $i$, we have $v\left(r_{i}\right)<k \leq v\left(r_{i+1}\right)$. Then HGCD-B returns $j=v\left(r_{i}\right), \alpha=2^{-j} r_{i}, \beta=2^{-j} r_{i+1}$, and a matrix $M$ such that

$$
\left(\begin{array}{l}
\alpha \\
\beta
\end{array}\right)=2^{-2 j} M\left(\begin{array}{l}
a \\
b
\end{array}\right) \text {. }
$$

We have $j<k$ and $j+v(\beta) \geq k$, and the size of the output is bounded by

$$
\begin{gathered}
\# M \leq\left\lfloor\frac{11(j+1)}{8}\right\rfloor \leq\left\lfloor\frac{11 k}{8}\right\rfloor, \\
\#(\alpha, \beta) \leq \begin{cases}\ell-\left\lfloor\frac{5 j-12}{8}\right\rfloor, & j>2, \\
\ell, & j=1,2 .\end{cases}
\end{gathered}
$$

These bounds are related to powers of the matrix $(0,2 ; 2,1)$, with eigenvalues $(1 \pm$ $\sqrt{17}) / 2$, with $\log _{2}[(1+\sqrt{17}) / 2]<11 / 8$. The HGCD-B function doesn't quite reduce the input numbers $a$ and $b$ to numbers $\alpha$ and $\beta$ of half the size, but the reduction is sufficient for GCD to terminate in subquadratic time.

The choice of $k$ implies $2 k+1 \leq \ell$, which guarantees that the quotients computed by HGCD-B are valid for any numbers for which $a$ and $b$ are the $\ell$ least significant bits. We can now describe Stehlé's and Zimmermann's binary recursive algorithm.

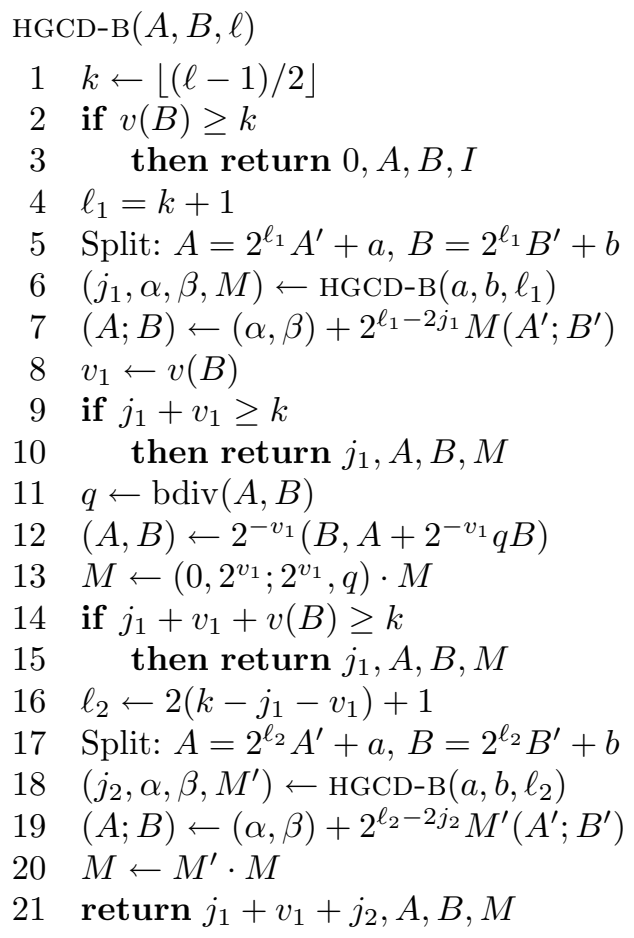

For analysis and further details, see [10].

\section{SCHÖNHAGE'S 1987 ALGORITHM}

Let $a$ and $b$ be two positive integers. Euclid's algorithm repeatedly replaces the larger number by the remainder when dividing the larger number by the smaller. 
We can decompose these steps further: Repeatedly replace the larger number by the difference between the numbers.

The Euclidean step corresponds to multiplying the vector $(a, b)^{T}$ by one of the matrices

$$
\left(\begin{array}{cc}
1 & -q \\
0 & 1
\end{array}\right) \text { or }\left(\begin{array}{cc}
1 & 0 \\
-q & 1
\end{array}\right)
$$

depending on which of $a$ and $b$ is largest. The decomposition of the division step as $q$ subtraction steps corresponds to the $q$ th power $(1,-q ; 0,1)=(1,-1 ; 0,1)^{q}$.

Note that these matrices all have determinant 1 , hence they have inverses with all-integer elements. The correctness proof for Euclid's algorithm can be generalized to the following important observation:

Lemma 3. If $a, b, \alpha$, and $\beta$ are integers, $M$ is $a 2 \times 2$ matrix, $\operatorname{det} M=1$, and

$$
\left(\begin{array}{l}
a \\
b
\end{array}\right)=M\left(\begin{array}{l}
\alpha \\
\beta
\end{array}\right)
$$

then $\operatorname{gcd}(a, b)=\operatorname{gcd}(\alpha, \beta)$.

Proof. Equation (2) directly implies that $\operatorname{gcd}(\alpha, \beta)$ divides both $a$ and $b$, and hence also $\operatorname{gcd}(a, b)$. By multiplying with $M^{-1}$ we get the other direction; $\operatorname{gcd}(a, b)$ divides $\operatorname{gcd}(\alpha, \beta)$.

We also need a bound for the matrix elements.

Lemma 4. If $a, b, \alpha, \beta>0, \#(a, b) \leq n$, \# $(\alpha, \beta)>s$, and equation (2) holds with some $M \geq 0$, then $\# M \leq n-s$. In fact, if $M=\left(u, u^{\prime} ; v, v^{\prime}\right)$, we also have $\#\left(u+u^{\prime}, v+v^{\prime}\right) \leq n-s$.

Proof. From the first row of equation (2) we get

$$
2^{n}>a=u \alpha+u^{\prime} \beta \geq u 2^{s}+u^{\prime} 2^{s}=2^{s}\left(u+u^{\prime}\right)
$$

and the result follows by dividing both sides by $2^{s}$. The same argument applies to the second row.

During the algorithm, we need a division operation $\operatorname{sdiv}(a, b, s)$ that never returns a too small "remainder". Input is a bit size $s$ and two positive integers $a$ and $b$ with $\#(a, b)>s$. Then sdiv produces the largest $q$ such that $q b<a$ and $\#(a-q b)>s$. It can be implemented using standard division as follows: First compute $q^{\prime}=\lfloor a / b\rfloor$. If $\#\left(a-q^{\prime} b\right)>s$, set $q=q^{\prime}$, otherwise, set $q=q^{\prime}-1$. Finally, return $q$ and $r=a-q b$.

After these preliminaries, we describe a generalized GCD function.

\subsection{The SGCD function.}

Lemma 5. Given a bit size $s \geq 0$ and two positive integers $a$ and $b$ with $\#(a, b)>s$, there exists integers $\alpha, \beta$, and a matrix $M$ such that

$$
\begin{aligned}
M & \geq 0, & \alpha, \beta & >0, \\
\left(\begin{array}{l}
a \\
b
\end{array}\right) & =M\left(\begin{array}{l}
\alpha \\
\beta
\end{array}\right), & \#(\alpha, \beta) & >s, \\
\operatorname{det} M & =1, & \#(\alpha-\beta) & \leq s .
\end{aligned}
$$

Existence follows from the following algorithm, using repeated division: 


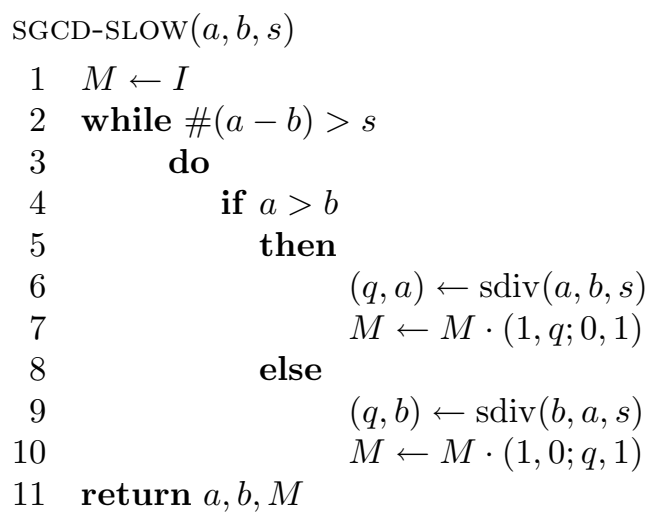

This is essentially Euclid's algorithm, but with a different stop condition. In fact, $\alpha$, $\beta$, and $M$ are determined uniquely by $a, b, s$, and the conditions of equation (3); this is not needed for the algorithm to work, but it is nevertheless proved in Appendix $\mathrm{A}$.

By Lemma 3, $\operatorname{gcd}(a, b)=\operatorname{gcd}(\alpha, \beta)$, and by Lemma 4. $\# M \leq \#(a, b)-s$. The SGCD function is a generalization of extended gcd, since for $s=0$, SGCD terminates with $\alpha=\beta=\operatorname{gcd}(a, b)$ and a matrix $M=\left(u, u^{\prime} ; v, v^{\prime}\right)$ such that $\operatorname{gcd}(a, b)=$ $v^{\prime} a-u^{\prime} b=-v a+u b$.

5.2. Subquadratic SGCD. The SGCD algorithm in Figure 5 is a step-by-step translation of Schönhage's algorithm for reduction of quadratic forms [8].

The correctness argument in 8 carries over directly, and will not be repeated here, except for explaining Step 8 which is the most subtle one.

Consider the case $p>0$. Before the adjustment, we have $\#(\alpha, \beta)>s^{\prime}, \#(\alpha-\beta) \leq$ $s^{\prime}$. We also have $p+s^{\prime}=s+1$ and a bound for the matrix elements, \#M $\leq$ $(n+s-p)-s^{\prime}=n-1$. Let $M=\left(u, u^{\prime} ; v, v^{\prime}\right)$, then the new adjusted values for $\alpha$ and $\beta$ are given by

$$
2^{p}\left(\begin{array}{l}
\alpha \\
\beta
\end{array}\right)+\left(\begin{array}{cc}
v^{\prime} & -u^{\prime} \\
-v & u
\end{array}\right)\left(\begin{array}{l}
A^{\prime} \\
B^{\prime}
\end{array}\right) .
$$

Consider the first row. For the first term, we have $\#\left(2^{p} \alpha\right)>p+s^{\prime}=s+1$. For the second term, we have $\#\left(v^{\prime} A^{\prime}-u^{\prime} B^{\prime}\right) \leq n-1+p=s$. Then

$$
2^{p} \alpha+\left(v^{\prime} A^{\prime}-u^{\prime} B^{\prime}\right) \geq 2^{s+1}-2^{s}=2^{s} .
$$

A similar inequality holds for $\beta$.

We also need an upper bound for $\#(\alpha-\beta)$ after adjustment. Let $d$ denote the difference $\alpha-\beta$ before the adjustment step, then $\# d \leq s^{\prime}$. After the adjustment, we get

$$
\begin{aligned}
|\alpha-\beta| & \leq 2^{p} d+\left|\left(v+v^{\prime}\right) a_{0}-\left(u+u^{\prime}\right) b_{0}\right| \\
& <2^{p+s^{\prime}}+2^{n-1+p}=2^{s+1}+2^{s}<2^{s+2} .
\end{aligned}
$$

To summarize, after Step 8 we have $\#(\alpha, \beta) \geq s, \#(\alpha-\beta) \leq s+2$.

The analysis for the running time in $[8$ also applies directly to SGCD. Let $T(n)$ denote the maximal running time for inputs with $s \leq 3 n$ and $\#(a, b) \leq n+s$. Then the argument in $[8$, shows that $T(n) \leq c \mu(n) \log n$.

When using SGCD to compute plain gcd, without co-factors, one disadvantage of the SGCD algorithm as described above is that it computes the matrix $M$ even though it is not needed. One important optimization is to note that if the caller 


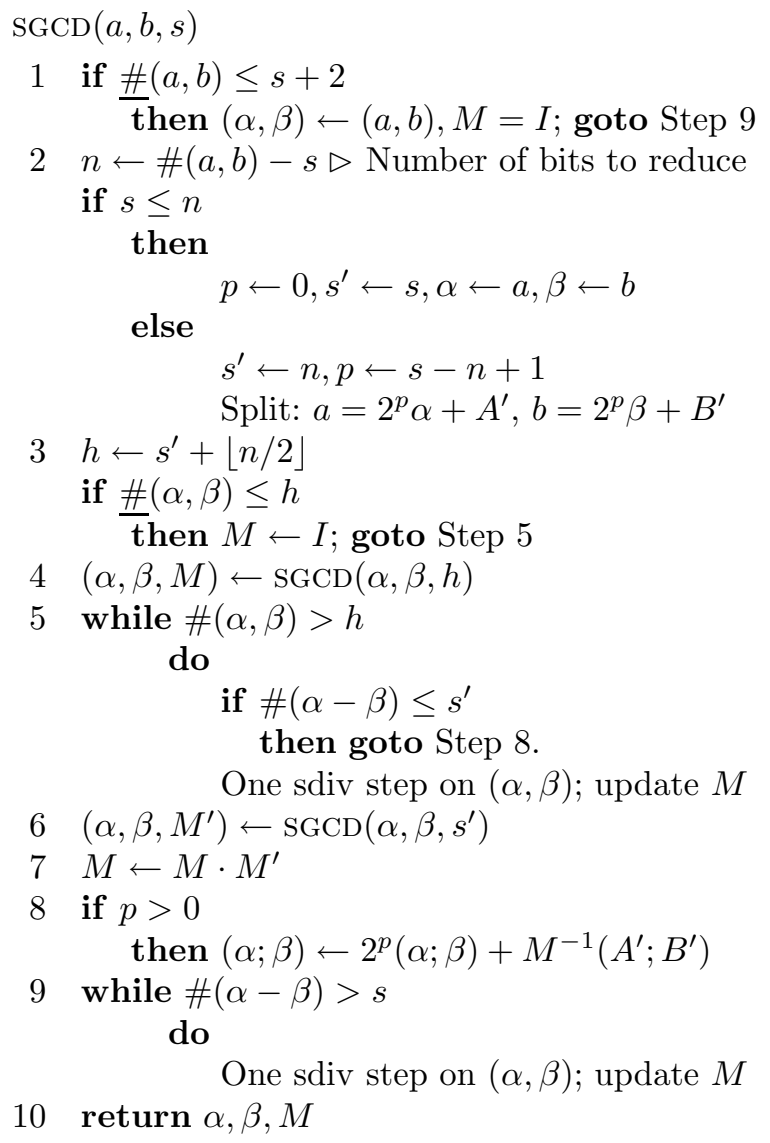

Figure 5. The SGCD algorithm

doesn't need $M$, and $p=0$, then it is not necessary to compute the product $M \cdot M^{\prime}$ in Step 7 or the two factors. Avoiding these unnecessary computations is essential for making the algorithm competitive, since it eliminates the final and largest matrix multiplication.

\section{A NEW HALF-GCD FUNCTION}

In this section, we reorganize Schönhage's 1987 algorithm to put it into half-gcd form. There are two main changes: The responsibility for the adjustment, Step 8 , is moved to the caller of the function, and the $s$ parameter is not passed freely, but is determined by input size. This makes the algorithm more streamlined and easier to compare to the other half-gcd algorithms, and the elimination of $s$ also makes the analysis of the running time more straightforward.

6.1. The HGCD-D function. In short:

$$
\operatorname{HGCD}-\mathrm{D}(a, b)=\operatorname{SGCD}(a, b,\lfloor \#(a, b) / 2\rfloor+1) .
$$

The inputs are two integers $a, b>0$. Put $n=\#(a, b)$ and $s=\lfloor n / 2\rfloor+1$, and assume $\#(a, b)>s$. Then HGCD-D returns two numbers $\alpha, \beta>0$, with $\#(\alpha, \beta)>s$, but 
$\#(\alpha-\beta) \leq s$, and a matrix $M \geq 0$ such that

$$
\left(\begin{array}{l}
a \\
b
\end{array}\right)=M\left(\begin{array}{l}
\alpha \\
\beta
\end{array}\right) \quad \operatorname{det} M=1 .
$$

The bound in Lemma 4 implies that $\# M \leq n-s<n / 2$. This choice of $s$ is just sufficient to make it useful to apply HGCD-D to the most significant part of two larger numbers. The following lemma plays the role of the adjustment Step 8 in Section 5.2 .

Lemma 6. Let $A$ and $B$ be two given numbers of size $N=\#(A, B)$, assume $0<p<N$. Split $A$ and $B$ into the least significant $p$ bits $A^{\prime}$ and $B^{\prime}$ and the $n=N-p$ most significant bits $a$ and $b$, so that $A=2^{p} a+A^{\prime}$ and $B=2^{p} b+B^{\prime}$. Also assume that \# $(a, b)>\lfloor n / 2\rfloor+1$, so that $\operatorname{HGCD}-\mathrm{D}(a, b)$ is well defined. Let $(c, d, M)=\operatorname{HGCD}-\overline{\mathrm{D}}(a, b)$, and form

$$
\left(\begin{array}{l}
C \\
D
\end{array}\right)=M^{-1}\left(\begin{array}{l}
A \\
B
\end{array}\right)=2^{p}\left(\begin{array}{l}
c \\
d
\end{array}\right)+M^{-1}\left(\begin{array}{l}
A^{\prime} \\
B^{\prime}
\end{array}\right) .
$$

Then $\underline{\#}(C, D)>p+\lfloor n / 2\rfloor$ and $\#(C-D) \leq p+\lfloor n / 2\rfloor+2$.

Proof. From the definition of HGCD-D, we have $\# M \leq n-s$, \# $(c, d)>s$, and $\#(c-d) \leq s$, where $s=\lfloor n / 2\rfloor+1$. Note that $s>n / 2>n-s$. Let $M^{-1}=$ $\left(v^{\prime},-u^{\prime} ;-v, u\right)$. In equation (4) we have

$$
\begin{aligned}
C & =2^{p} c+v^{\prime} A^{\prime}-u^{\prime} B^{\prime} \\
& >2^{p} 2^{s}-2^{n-s} 2^{p} \geq 2^{p} 2^{s-1}=2^{p+\lfloor n / 2\rfloor} .
\end{aligned}
$$

Then $\# C>p+\lfloor n / 2\rfloor$ as claimed, and the same holds for $D$. Next,

$$
\begin{aligned}
|C-D| & \left.=\mid 2^{p}(c-d)+\left(v+v^{\prime}\right) A^{\prime}-\left(u+u^{\prime}\right) B^{\prime}\right) \mid \\
& <2^{p} 2^{s}+2^{n-s} 2^{p} \leq 2^{p+s+1}=2^{p+\lfloor n / 2\rfloor+2} .
\end{aligned}
$$

6.2. Subquadratic HGCD-D. The recursive algorithm in Figure 6 computes HGCDD in subquadratic time. The following lemma explains the most essential details:

Lemma 7. In the two recursive calls in Step 6 and 17 of the HGCD-D algorithm, we have

$$
\#(a, b) \leq\lceil N / 2\rceil .
$$

The loops at Step 9 and Step 20 are executed at most 4 times each.

Proof. Consider the two recursive calls in turn. For the first call in Step [6, it is clear that $n_{1}=\lceil N / 2\rceil$. We have

$$
p_{1}+\left\lfloor n_{1} / 2\right\rfloor=\lfloor N / 2\rfloor+\lfloor(N+1) / 4\rfloor=\lfloor 3 N / 4\rfloor
$$

and \# $(a, b)=\#(A, B)-p_{1}>\left\lfloor n_{1} / 2\right\rfloor+2$, so that HGCD-D $(a, b)$ is well defined. By Lemma 6, the new $A$ and $B$ after the call satisfy $\#(A-B) \leq\lfloor 3 N / 4\rfloor+2$, and after at most two sdiv steps, $\#(A, B) \leq\lfloor 3 N / 4\rfloor+2$. Then at most two additional steps are needed to reduce the size one more bit (two Euclid steps using standard division reduces the size by at least one bit. The remainders produced by sdiv differ from the Euclid remainders only when one of the Euclid remainders is of size $S$ or smaller, and in this case, the HGCD-D algorithm terminates with no further steps of any kind). 


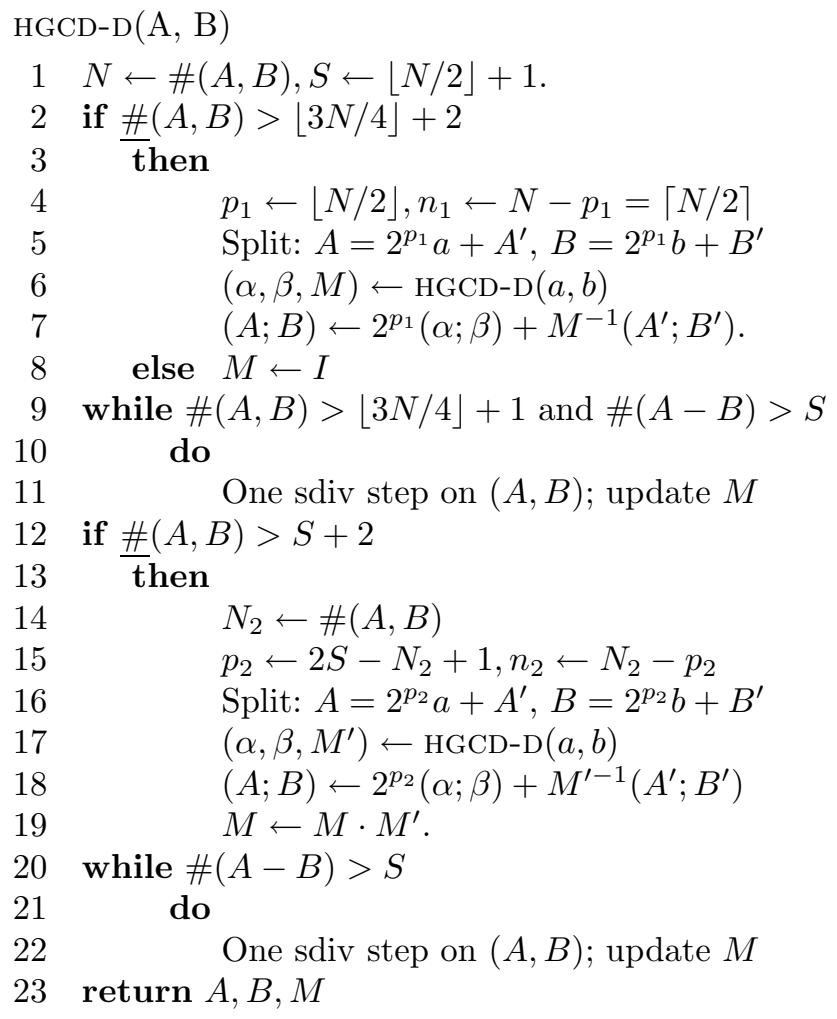

Figure 6 . The HGCD-D algorithm.

For the second recursive call, Step 17, first note that $n_{2}=2\left(N_{2}-S\right)-1$. The bound $N_{2} \leq\lfloor 3 N / 4\rfloor+1$ implies $n_{2}=2\left(N_{2}-S\right)-1 \leq 2(\lfloor 3 N / 4\rfloor-\lfloor N / 2\rfloor)-1 \leq$ $\lceil N / 2\rceil-1$.

Next, $\left\lfloor n_{2} / 2\right\rfloor=N_{2}-S-1$, and since \# $(a, b)>S+2-p_{2}=N_{2}-S+1=\left\lfloor n_{2} / 2\right\rfloor+2$, $\operatorname{HGCD}-\mathrm{D}(a, b)$ is well defined. We also get

$$
p_{2}+\left\lfloor n_{2} / 2\right\rfloor=\left(2 S-N_{2}+1\right)+\left(N_{2}-S-1\right)=S .
$$

Then Lemma 6 implies that after the call, the new $A$ and $B$ satisfy $\#(A-B) \leq$ $S+2$. It then takes at most four sdiv steps until the algorithm terminates with $\#(A-B) \leq S$.

Lemma 8. The above algorithm runs in time $T(n)=c \mu(n) \log n$, where $\mu(n)$ is the time for one multiplication or division of n-bit numbers. With FFT-based multiplication, $T(n)=O\left(n(\log n)^{2} \log \log n\right)$.

Proof. Let $T(n)$ denote the maximum running time of the HGCD-D algorithm, with input $a$ and $b$ of size $n$. Then $T(n) \leq 2 T(\lceil n / 2\rceil)+c \mu(n)$, which implies $T(n) \leq$ $c \mu(n) \log n$.

All the considered subquadratic GCD algorithms have the same asymptotic running time, but they may differ by constant factors. 
6.3. The need for backup steps. The difference between HGCD-Q and HGCD-D, i.e. between Schönhage's 1971 algorithm and the new algorithm, is quite subtle. The difference is in the definition of a correct reduction, and in the correctness and stop conditions that follow from that.

The correctness of HGCD-Q depends on the quotient sequence being correct. In fact, the HGCD-Q algorithm is expected to produce a quotient sequence that is the prefix of the continued fraction expansion for any $x$ in the interval $A /(B+1)<$ $x<(A+1) / B$. It seems that to achieve this, the backup steps are essential.

Let's consider an example where HGCD-Q without backup logic returns an incorrect result: $A=858824$ and $B=528747$. Then $n=20, m=10, \operatorname{gcd}(A, B)=1$ and the quotient sequence is $(1,1,1,1,1,1,20,1,1,3,3,5,8,3)$. The HGCD-Q algorithm reduces these numbers as follows:

\begin{tabular}{cc} 
Input & Output \\
858824,528747 & \\
\hline First rec. call & 128,66 \\
838,516 & $(2,-3 ;-3,5)$ \\
\hline Loop & 64144,3119 \\
131407, 67263 & $(5,-8 ;-8,13)$ \\
\hline Second rec. call & 129,65 \\
4009,194 & $(1,-20 ;-1,21)$ \\
\hline Without backup & 1764,1355 \\
& $(165,-268 ;-173,281)$
\end{tabular}

The final remainders, $r_{0}=1764$ and $r_{1}=1355$, violate both versions of Jebelean's criterion, since $r_{0}-r_{1}=409<2^{m}$, but $v_{2}-v_{1}=549$. For any $p \geq 3$, let $A^{\prime}=1$ and $B^{\prime}=2^{p}-1$; then the numbers $2^{p} A+A^{\prime}$ and $2^{p} B+B^{\prime}$ have a quotient sequence that starts with $(1,1,1,1,1,1,20,2)$, for example, $(8 A+1,8 B+7)=$ $(6870593,4229983)$ have the quotient sequence $(1,1,1,1,1,1,20,2,53,4,2,12,29)$. This shows that the backup steps in the HGCD-Q algorithm are essential for correctness; the final quotient must be discarded.

The stop and correctness conditions for HGCD-Q are related to Jebelean's criterion. We can only be sure that a quotient $q_{k}$ is correct if the remainder after the next division step, $r_{k+2}=r_{k}-q_{k+1} r_{k+1}$, is large. And the stop condition checks if the remainder after yet another division, $r_{k+3}=r_{k+1}-q_{k+2} r_{k+2}$, is small.

The new algorithm, HGCD-D, uses a relaxed notion of a correct reduction. It does not pay attention to the quotient sequence, instead its correctness is based on the more general Lemma 3 . This relaxation makes it possible to use simpler correctness and stop conditions: A co-factor matrix is correct if the two corresponding remainders $r_{k}$ and $r_{k+1}$ are large, and the stop condition checks if $\left|r_{k}-r_{k+1}\right|$ is small.

\section{Evaluation}

The GCD algorithms were implemented in the mpn layer in GMP [1, and compared with respect to running time and implementation complexity. We compare the following algorithms: 
TABLE 1. Threshold values used for the various algorithms, tuned for an AMD Duron.

\begin{tabular}{l|rr} 
Algorithm & \multicolumn{2}{|c}{ Thresholds } \\
& HGCD & GCD \\
\hline mpn_rgcd & 191 & 951 \\
mpn_bgcd & 133 & 1015 \\
mpn_sgcd & 95 & 1193 \\
mpn_ngcd & 159 & 866
\end{tabular}

mpn_gcd: Kenneth Weber's accelerated GCD algorithm, shipped in GMP-4.1.4, falling back to Lehmer's algorithm for small inputs 2

mpn_rgcd: Schönhage's 1971 algorithm, as described in Section 3.

mpn_bgcd: Stehlé's and Zimmermann's recursive binary GCD algorithm, as described in Section 4 .

mpn_sgcd: Schönhage's 1987 algorithm, as described in Section 5 The implementation avoids computing unneeded matrices.

mpn_ngcd: GCD based on the HGCD-D algorithm, as described in Section 6 .

As far as possible, the implementation avoids splitting numbers at arbitrary bit boundaries, and instead works with word boundaries.

7.1. Threshold values. All the subquadratic algorithms use quadratic base cases in two ways, in the half-gcd and full-gcd functions. The use of GCD-THRESHOLD has already been described in Section 2. For the HGCD-style functions, when the input size is below HGCD-THRESHOLD, the functions stop chopping numbers in half, and instead repeatedly chop off two words at a time, using a HGCD function specialized for input size of two words, and producing a transformation matrix with single word elements. For the left-to-right algorithms, this is analogous to Lehmer's algorithm. The input size and the thresholds are expressed in number of words, where a word is 32 bits on the architecture used for the evaluation.

The optimal threshold values depend on the architecture. Table 1 gives the thresholds used on an AMD Duron processor. These values are tuned to get close to optimal.

7.2. Performance. Figure 7 shows the running time for inputs ranging from 10 words (320 bits) to 110000 words (3400000 bits), evaluated on an $1.4 \mathrm{GHz}$ AMD Duron (a fairly low-end PC). On this logarithmic scale, all the subquadratic algorithms are very close, and they start to pay off for sizes around 1000 words and larger; at 10000 words, the subquadratic algorithms are about twice as fast as Weber's accelerated GCD algorithm, and for 100000 words they are about 30 times faster.

The running time for the different subquadratic algorithms differs by constant factors, which can be seen if we focus on input sizes of a few thousand words. The running times on the same processor are shown in Figure 8, using a linear scale for both input size and running time.

\footnotetext{
${ }^{2}$ GMP-4.1.4 uses the binary algorithm for small inputs, but it turns out that Lehmer's algorithm is faster for numbers up to a few dozen words, so that is what is used in this evaluation.
} 


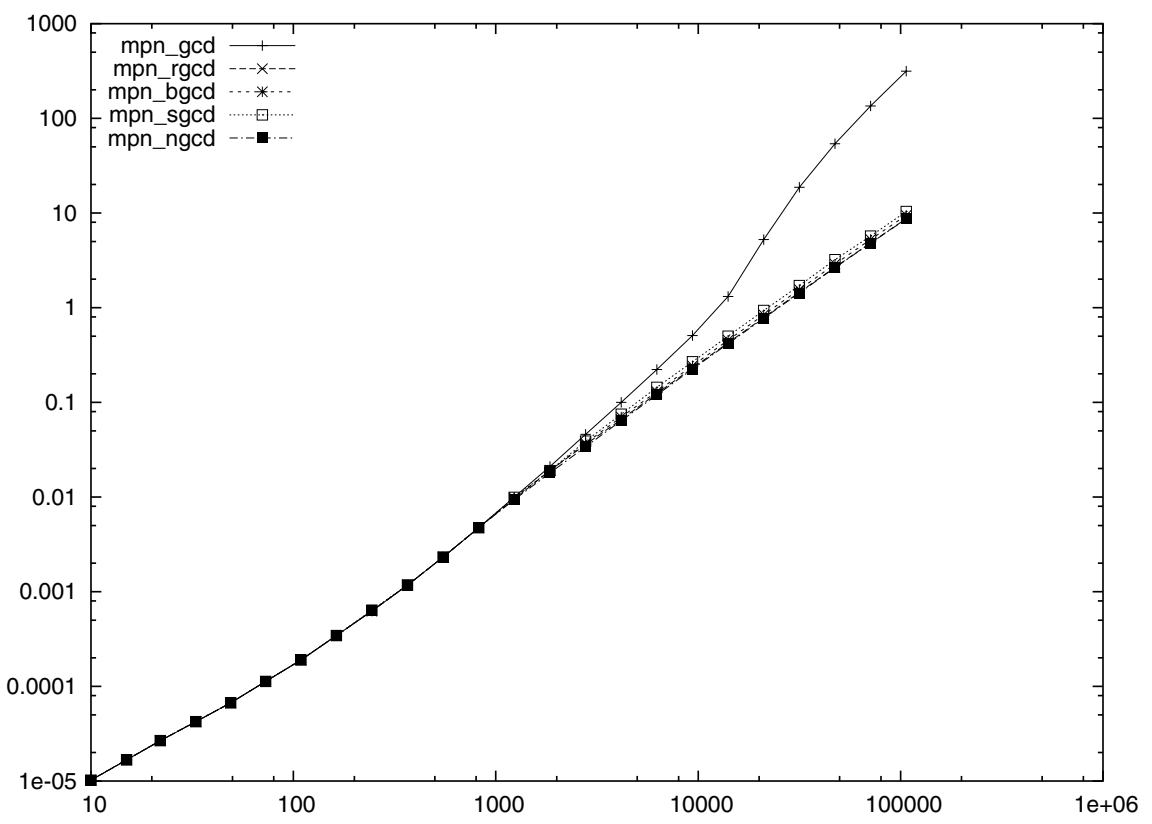

Figure 7. Running for large inputs on AMD Duron. Both sizes (in words) and running times (in second) are shown in logarithmic scale. The mpn_gcd function is much slower than the subquadratic algorithms, which all look very similar.

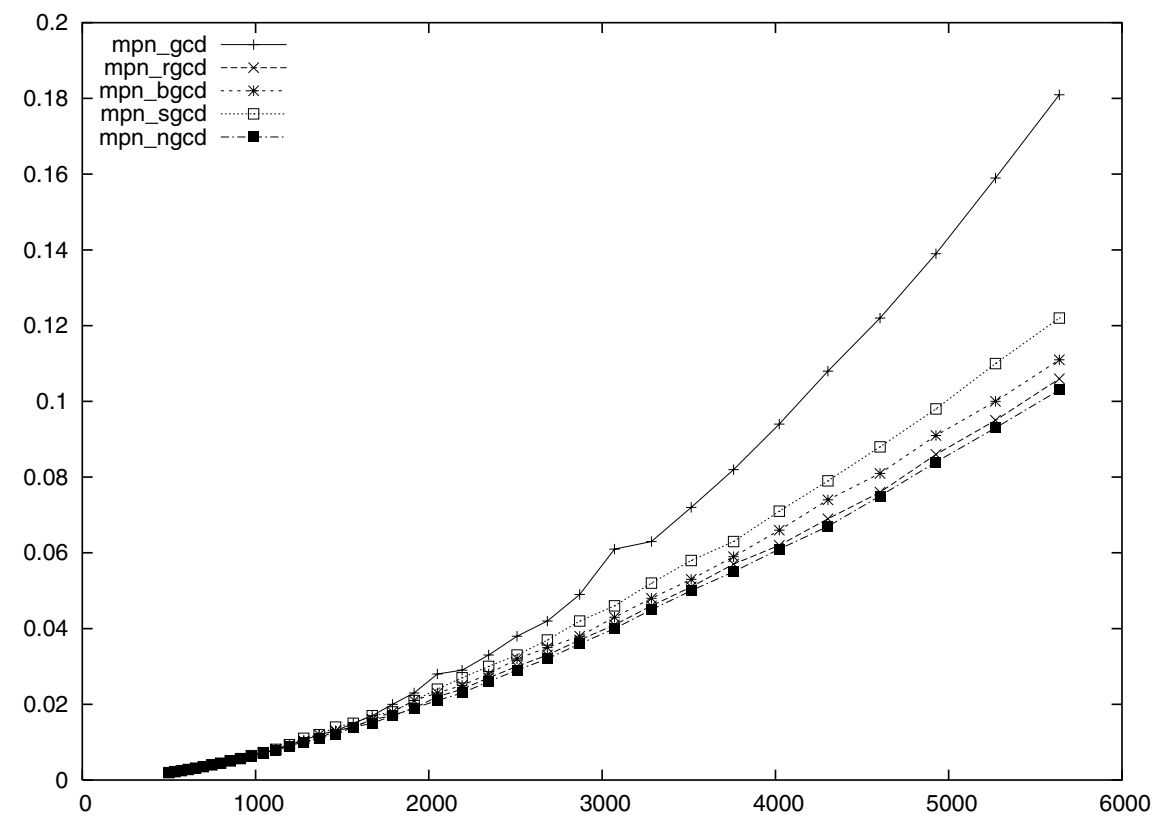

Figure 8. Running time for medium size input, on an AMD Duron. mpn_gcd is slowest, as expected, followed by mpn_sgcd, and mpn_bgcd. The new algorithm, mpn_ngcd, is fastest, but with a very small margin to mpn_rgcd. 
TABLE 2. Number of non-comment lines of code, and cyclomatic complexity, for the subquadratic GCD algorithms.

\begin{tabular}{l|rr} 
Algorithm & \# lines & $\begin{array}{r}\text { Cyclomatic } \\
\text { complexity }\end{array}$ \\
\hline mpn_rgcd & 1967 & 292 \\
mpn_bgcd & 1348 & 206 \\
mpn_sgcd & 760 & 144 \\
mpn_ngcd & 733 & 133
\end{tabular}

We see the quadratic behavior of the mpn_gcd function, as expected. The binary algorithm is $5-10 \%$ slower than Schönhage's 1971 algorithm. It is often hard to explain performance differences between algorithms that have the same asymptotic running time and differ only by a constant factor. The binary algorithm is simpler in structure, but works with slightly larger numbers. Where an application of the HGCD-Q function in Schönhage's algorithm reduces $n$-bit numbers to a size close to $n / 2$ bits, an application of the HGCD-B function in the binary algorithm can produce numbers of size close to $11 n / 16$. Other factors that need investigation are the average number of quotients and the average number of quotients that can be represented by a single word matrix $M$.

Also seen in Figure 8, implementing Schönhage's 1987 algorithm as described in Section 5results in a subquadratic algorithm that is slower than both the binary recursive algorithm and Schönhage's 1971 algorithm. However, when reorganized into half-gcd form, performance is significantly improved, and the algorithm mpn_ngcd is slightly faster than mpn_rgcd.

7.3. Complexity. Of the compared algorithms, Schönhage's 1971 algorithm seems to be the most complex. Comparisons using popular code complexity measures, such as number of lines and McCabe's cyclomatic complexity, support this impression. See Table 2 for a detailed comparison. In our implementation, Schönhage's 1971 algorithm, mpn_rgcd, is more than twice as large as the new mpn_ngcd algorithm.

When reading the line counts, one should keep in mind that the implementation environment is quite low-level. The code uses library functions for basic arithmetic on large non-negative numbers, but both signs and storage sizes have to be kept track of explicitly.

In this comparison, the binary recursive algorithm, mpn_bgcd, turns out to be more complex than mpn_ngcd. This is a little surprising, since the algorithm as described in Section 4 is so straightforward. Some factors that contribute to the measured implementation complexity of the binary recursive algorithm are:

- The bdiv function is not part of the standard arithmetic library.

- It is essential to the binary recursive algorithm to use signed quotients and matrix elements.

- Bit shift logic in the adjustment step, depending on the $j$ returned by the recursive HGCD-B calls.

\section{Conclusions}

We describe a new subquadratic GCD algorithm building on Schönhage's work in the late 1980s. This is shown to have the same asymptotic complexity as both 
Schönhage's algorithm from 1971, and the binary recursive algorithm by Stehlé and Zimmermann. The new algorithm is slightly faster and much simpler to implement. Hopefully, this can open the door for wider use of subquadratic GCD in practice.

\section{Appendix A. Uniqueness of $M$}

To prove that $\alpha, \beta$, and $M$, the output of $\operatorname{SGCD}(a, b, s)$, is uniquely determined by $a, b$, and $s$, we first show that the matrix $M$ has a particular factorization.

Lemma 9. If $M \geq 0$ and $\operatorname{det} M=1$, then $M$ can be factorized as a product where each factor is either $(1,1 ; 0,1)$ or $(1,0 ; 1,1)$.

Proof. We use induction over the sum of matrix elements. Let $M=\left(u, u^{\prime} ; v, v^{\prime}\right)$ and $n=u+u^{\prime}+v+v^{\prime}$. If $n=2$, then the identity matrix is the only one that has unit determinant and non-negative elements, and it has a trivial factorization.

Make the induction assumption that all matrices with element sum smaller than $n$ have a factorization of the required form.

For $n>2$, we have a few different cases, depending on the relative order of the matrix elements $u, u^{\prime}, v$, and $v^{\prime}$. If one row is elementwise larger than the other, e.g., $u \geq v$ and $u^{\prime} \geq v^{\prime}$, then

$$
M=\left(\begin{array}{ll}
1 & 1 \\
0 & 1
\end{array}\right)\left(\begin{array}{cc}
u-v & u^{\prime}-v^{\prime} \\
v & v^{\prime}
\end{array}\right)
$$

where the matrix on the right is non-negative, has unit determinant, and a reduced element sum $n-u^{\prime}-v^{\prime}<n$ (note that $\operatorname{det} M=1$ implies that $u$ and $v^{\prime}$ are nonzero). Hence, by induction, it has a factorization of the required form. Similarly, if $u \leq v$ and $u^{\prime} \leq v^{\prime}$, we can take out the factor $(1,0 ; 1,1)$.

If neither row is larger than the other, two cases remain: If $u>v$ and $u^{\prime}<v^{\prime}$, then $1=u v^{\prime}-u^{\prime} v \geq v^{\prime}(u-v) \geq 1$, which implies that $v^{\prime}=u-v=1$, and leads to $M=I$, contradicting $n>2$. On the other hand, if $u<v$ and $u^{\prime}>v^{\prime}$, then $1=u v^{\prime}-u^{\prime} v \leq v\left(v^{\prime}-u^{\prime}\right) \leq 0$, a contradiction.

We can now prove that $\alpha, \beta$, and $M$ are uniquely determined by $a, b, s$, and the conditions of equation (3). This result follows from the next lemma, specialized to $k=2^{s}$.

Lemma 10. If $k>0$ and $a, b \geq k$ are given, then $\alpha, \beta$, and $M$ are uniquely determined by the requirements $(a ; b)=M(\alpha ; \beta), M \geq 0$, $\operatorname{det} M=1, \alpha \geq k$, $\beta \geq k$, and $|\alpha-\beta|<k$.

Proof. From the previous lemma, we know that either $M=I, M=(1,1 ; 0,1) M^{\prime}$, or $M=(1,0 ; 1,1) M^{\prime}$, where $M^{\prime} \geq 0$ and $\operatorname{det} M^{\prime}=1$. When $M \neq I$, let $\left(a^{\prime} ; b^{\prime}\right)=$ $M^{\prime}(\alpha ; \beta)$, then $a^{\prime}, b^{\prime} \geq k$ and either $\left(a^{\prime}, b^{\prime}\right)=(a-b, b)$ or $\left(a^{\prime}, b^{\prime}\right)=(a, b-a)$.

We use induction on $n=\max (a, b)$.

If $|a-b|<k$, in particular if $a=b$, then we must have $M=I$, since otherwise, either $a^{\prime}$ or $b^{\prime}$ equals $|a-b|<k$, which contradicts $a^{\prime}, b^{\prime} \geq k$.

If $|a-b| \geq k$, then we must have $M \neq I$. If $a>b$, then we must have $\left(a^{\prime} ; b^{\prime}\right)=$ $(a-b ; b)$, so that $\max \left(a^{\prime}, b^{\prime}\right)<a=n$. By induction, $\alpha, \beta$, and $M^{\prime}$ are uniquely determined by $a^{\prime}, b^{\prime}$, and $k$, and then $M=(1,1 ; 0,1) M^{\prime}$. The case $a<b$ is similar. 


\section{ACKNOWLEDGEMENTS}

The author wishes to thank Bradley Lucier, for pointing out the importance of Schönhage's 1991 paper, and for helpful discussions on how to implement and optimize it, Torbjörn Granlund, for writing GMP and for his zealous testing, and Damien Stehlé, for helping me understand the binary recursive algorithm, and for many helpful comments during the work with this paper.

\section{REFERENCES}

[1] Torbjörn Granlund, GNU multiple precision arithmetic library, version 4.1.4, September 2004, http://swox.com/gmp

[2] Tudor Jebelean, A double-digit Lehmer-Euclid algorithm for finding the GCD of long integers, Journal of Symbolic Computation 19 (1995), no. 1-3, 145-157. MR1339115 (96h:11128)

[3] Donald E. Knuth, The analysis of algorithms, Actes du Congrés International des Mathématiciens (1970), 269-274. MR0423865 (54:11839)

[4] D. H. Lehmer, Euclid's algorithm for large numbers, American Mathematical Monthly 45 (1938), 227-233. MR.1524250

[5] Bradley Lucier, Personal communication, 2005.

[6] Victor Y. Pan and Xinmao Wang, Acceleration of Euclidean algorithm and extensions, ISSAC '02: Proceedings of the 2002 International Symposium on Symbolic and Algebraic Computation (New York, NY, USA), ACM Press, 2002, pp. 207-213. MR.2035251 (2005a:68101)

[7] Arnold Schönhage, Schnelle Berechnung von Kettenbruchentwicklungen, Acta Informatica 1 (1971), 139-144.

[8] - Fast reduction and composition of binary quadratic forms, Proc. of Intern. Symp. on Symbolic and Algebraic Computation (Bonn) (S. M. Watt, ed.), ACM Press, 1991, pp. 128133.

[9] Jonathan P. Sorenson, Two fast GCD algorithms, Journal of Algorithms 16 (1994), no. 1, 110-144. MR1251841 (94k:11135)

[10] Damien Stehlé and Paul Zimmermann, A binary recursive GCD algorithm, ANTS-VI (Burlington) (D. Buell, ed.), LCNS, vol. 3076, Springer-Verlag, June 2004. MR2138011 (2006e:11194)

[11] J. Stein, Computational problems associated with Racah algebra, Journal of Computational Physics 1 (1967), no. 3, 397-405.

[12] Klaus Thull and Chee K. Yap, A unified approach to HGCD algorithms for polynomials and integers, 1990, Manuscript. Available from http://cs.nyu.edu/cs/faculty/yap/papers

[13] Kenneth Weber, The accelerated integer GCD algorithm, ACM Transactions on Mathematical Software 21 (1995), 111-122. MR1365811 (96h:68084)

[14] André Weilert, Ein schneller algorithmus zur berechnung des quartischen restsymbols, Diplomarbeit, Math. Inst. der Univ., Bonn, 1999.

[15] _ Asymptotically fast GCD computation in Z[i], ANTS-IV: Proc. of Fourth Intern. Symp. Algorithmic Number Theory (Leiden), LNCS, no. 1838, Springer-Verlag, July 2000, pp. 595-613. MR:1850636 (2002k:11226)

Automatic Control, KTH, SE-100 44, Sweden

E-mail address: nisse@lysator.liu.se 